\title{
Niveles de citoquinina y su efecto sobre la producción de caigua (Cyclanthera pedata L.).
}

Cytokinin levels and their effect on the production of caigua (Cyclanthera pedata L.).

Jesús Adeudato Silva Antaurco, Dante Daniel Cruz Nieto, Francisco Espinoza Montesinos ${ }^{1}$, José Antonio Legua Cárdenas ${ }^{2}$,Jaime Ulices Romero Menacho²

\section{RESUMEN}

Objetivo: Determinar la dosis adecuada de citoquinina que tenga influencia en el rendimiento y calidad de fruto, Métodos: Se empleó el diseño experimental de bloque completamente al azar con 5 tratamientos y 4 repeticiones, El ensayo tuvo el testigo (Sin citoquinina), y las siguientes dosis; 50, 100, 150 y $200 \mathrm{ml} / 200 \mathrm{~L}$ de agua, dichas dosis se aplicaron a los 20 y 60 días después de la siembra, Se evaluaron las características físicas, químicas y se hizo el respectivo análisis económico, Los datos fueron procesados con el análisis de Varianza y la Prueba de Duncan, Resultados: La dosis $\mathrm{T}_{3}$ con $100 \mathrm{ml}$ de citoquininas obtuvo los resultados: Cantidad de frutos por planta 26, Peso promedio por planta 2,28 kg, peso de un fruto por planta 112,16 g y rendimiento comercial 30,60 Tn/ha, En el análisis químico se evidenció una baja concentración del macronutriente potasio con $6,80 \%$, los demás elementos estuvieron en niveles normales y en el análisis económico esta misma dosis sobresalió en utilidad con S/ 11695 Nuevos Soles, Conclusíon: La dosis de $\mathrm{T}_{3}$ con $100 \mathrm{ml}$ de citoquinina por $200 \mathrm{~L}$, sobresalió en rendimiento comercial, calidad y utilidad, siendo estos resultados favorable para los agricultores de la zona.

Palabras clave: Caigua, citoquinina, dosis, rendimiento, rentabilidad.

\section{ABSTRACT}

Objective: To determine the appropriate dose of cytokinin that has an influence on the yield and fruit quality, Methods: The Experimental Design of Completely Random Block was used with 5 treatments and 4 repetitions, The trial had the control (Without cytokinin), and the following doses; 50, 100, 150 and $200 \mathrm{ml} /$ 200 I of water, These doses were applied at 20 and 60 days after sowing, The physical and chemical characteristics were evaluated and the respective economic analysis was made, The data were processed with the Analysis of Variance and the Duncan Test, Results: The $T_{3}$ dose with $100 \mathrm{ml}$ of cytokinins obtained the results: Number of fruits per plant 26, Average weight per plant 2,28 kg, weight of one fruit per plant $112,16 \mathrm{~g}$ and commercial yield 30,60 Tn/ha, The chemical analysis showed a low concentration of macronutrient potassium with $6,80 \%$, the other elements were at normal levels and in the economic analysis this same dose stood out in usefulness with S/ 11695 Nuevos Soles, Conclusion: The dose of $\mathrm{T}_{3}$ with $100 \mathrm{ml}$ of cytokinin per $200 \mathrm{I}$, excelled in commercial performance, quality and usefulness, these results being favorable for farmers in the area.

Keywords: Caigua, cytokinin, dose, performance, profitability.

\footnotetext{
'Universidad Nacional Santiago Antunez de Mayolo. Ancash, Perú.

${ }^{2}$ Universidad Nacional José Faustino Sánchez Carrión. Huacho, Perú
} 


\section{INTRODUCCIÓN}

La caigua, es un cultivo de origen peruano que se siembra en la costa, sierra y selva, principalmente en las regiones de Lima, La Libertad, Lambayeque, Cajamarca, Ancash, Amazonas y otras, Es catalogada por su efectividad terapéutica pues esta planta constituye un factor muy importante para la solución de varios problemas relacionados con la obesidad (triglicéridos y colesterol) (Campos y Quintana, 2013). Por esta razón es apreciado en el consumo diario y la comercialización sigue aumentando en el mercado nacional e internacional entre ellos Estados Unidos y Europa.

Por lo consiguiente, la citoquinina regula numerosos procesos biológicos y fisiológicos en los vegetales, entre ellos controla la citocinesis, participa en la síntesis de proteínas, disminuye la dominancia apical, permitiendo el crecimiento de ramificaciones laterales e iniciación de yemas, acelera la germinación e influye sobre el transporte de nutrientes y metabolitos, retrasando la senescencia de flores, frutos y hojas, posee acción promotora en la translocación de nutrientes (Salisbury y Ross, 1994).

Cabe mencionar que se ha realizado investigaciones sobre el efecto de hormonas, según Cadenas (2015) se evaluó en diez y nueve tratamientos derivados de la combinaciones de seis dosis de hormonas y 3 períodos de aplicación más un testigo, se midió el rendimiento de esquejes (Penstemon campanulatus) exportables por maceta, días de ciclo de crecimiento de un esqueje. Se determinó que el tratamiento que obtuvo mejor resultado fue aplicando $0,0025 \mathrm{~g} / \mathrm{L}$, $\left(T_{9}\right)$ con 5,57 esquejes/maceta, con el cual obtuvo el mayor rendimiento en comparación a 0,0005, y 0,$001 ; 0,01$ y $0,025 \mathrm{~g} / \mathrm{L}$.

Arohuanca (2015), investigó diferentes dosis de bioestimulante a base de citoquinina en el cultivo de vainita que utilizó como material dos variedades (Hade y Derby) y el bioestimulante a base de citoquininas. Los resultados evidenciaron la doble dosis comercial y la variedad Hade, respondieron mejor, donde el mayor rendimiento se obtuvo con la doble dosis comercial con $11,60 \mathrm{t} / \mathrm{ha}$; en segundo lugar se ubica la dosis comercial con 10,55 t/ha; por el cual superó a la variedad Derby quedando en tercer lugar con la doble de dosis comercial con 8,20 t/ha.

Bahamonde (2006), en su investigación respuesta de tres cultivares (Florex Gold, Sensation, Pot of Gol') y un compuesto (mezcla de variedades que ha sido denominado Santa Rosa), de nombre científico Zantedeschia spp de color amarillo, Para ello se usaron dos productos de
Promalina $(5,28 \mathrm{ml} / \mathrm{L})$ y Cytokin $(10 \mathrm{ml} / \mathrm{L})$, Los resultados fueron mayor diámetro de Cytokin a $1,90 \mathrm{~cm}$ y 1,70 en hibrido de Santa Rosa, número de flores 1,5 mayor con Cytokin $(10 \mathrm{ml} / \mathrm{L})$, respecto a 1,25 flor de testigo, longitud de tallo 54 $\mathrm{cm}$ con Cytokin $(10 \mathrm{ml} / \mathrm{L})$ mayor al testigo $45 \mathrm{~cm}$, Los resultados indican el desarrollo vegetativo afectó la aplicación de Cytokin a diferencia de Promalina.

Villatoro (2014) evaluaron diferentes dosis de CPPU (Citoquininas) en aplicaciones sobre el cuaje de la flor de sandía, La investigación se llevó a cabo en condiciones de campo abierto, Los tratamientos fueron de 25, 50, 100 y 150 ppm de CPPU, aplicándose en diferente número de aplicaciones $(30,33$ y 36 días después del trasplante), Los análisis mostraron que con las aplicaciones de CPPU variaron las flores de sandía, en su rendimiento por hectárea alcanzó a $27,61 \mathrm{tn} / \mathrm{ha}$, disminuyendo con $150 \mathrm{ppm}$ a 27,47 tn/ha, Lo que concluye que baja significativamente a $150 \mathrm{ppm}$ en tres aplicaciones.

La Provincia de Barranca, también tiene condiciones agroecológicas apropiadas para el desarrollo de este cultivo, sembrándose en los meses de julio y agosto, se fertiliza con nutrientes sintéticos y productos naturales, como es el caso del uso de bioestimulante (Citoquinina) que se emplean continuamente; sin embargo no se conocía la dosis adecuada, Por este motivo, se realizó la investigación, con el fin de determinar la dosis óptima, para obtener buen rendimiento y calidad de fruto, que favorezca a los agricultores del Sector de Santa Elena.

\section{MATERIALY MÉTODOS}

El presente trabajo de investigación tiene como fin determinar que dosis de citoquinina del producto comercial Antesis Plus influye en el rendimiento de caigua (Cyclantera pedata L.) variedad pais, para ello se realizó este experimento, el mismo que servirá como diagnóstico para los agricultores del sector Santa Elena. Cabe mencionar que se trata de una investigación aplicada, pues los resultados obtenidos son replicables y serán aplicados por los agricultores de la zona.

En el desarrollo de la investigación se delimitó el área experimental, se empleó el modelo estadístico de Diseño de Bloques Completamente al Azar con cinco tratamientos incluido el testigo $\left(T_{1}\right.$ con $0, T_{2}$ con $50, T_{3}$ con $100, T_{4}$ con 150 y $T_{5}$ con $200 \mathrm{ml} / 200$ I,) y cuatro repeticiones, Las aplicaciones fueron a los 20 y 60 días después de la siembra en la etapa vegetativa y floracion. En cuanto al análisis estadístico se operó los datos con el análisis de varianza y la prueba de Duncan, también se obtuvo los resultados de análisis 
químico y económico. En el siguiente cuadro se muestran las diferentes dosis de citoquinina
(Antesis Plus), que se aplicaron al cultivo de caigua a los 20 y 60 días después de la siembra (Tabla 1).

Tabla 1. Aplicaciones de citoquinina (Antesis plus), al cultivo de caigua

\begin{tabular}{ccccc} 
& & \multicolumn{2}{c}{ Días de aplicación } & \\
\cline { 3 - 4 } Tratamiento & Dosis Ha & $\mathbf{2 0}$ días/ cil & $\mathbf{6 0}$ días/ $\mathbf{2}$ cil & Total /ha \\
\cline { 3 - 4 } $\mathrm{T}_{1}$ & 0 & 0 & 0 & 0 \\
$\mathrm{~T}_{2}$ & 50 & 50 & 100 & 150 \\
$\mathrm{~T}_{3}$ & 100 & 100 & 200 & 300 \\
$\mathrm{~T}_{4}$ & 150 & 150 & 300 & 450 \\
$\mathrm{~T}_{5}$ & 200 & 200 & 400 & 600 \\
\hline
\end{tabular}

Se emplearon las mismas labores agronómicas para todas las parcelas, variando solamente las dosis aplicadas de citoquinina (Antesis plus), cabe mencionar que se instalaron estacas en cada parcela, con el fin de que haya buena aeración, buen monitoreo para el control de plagas y enfermedades.

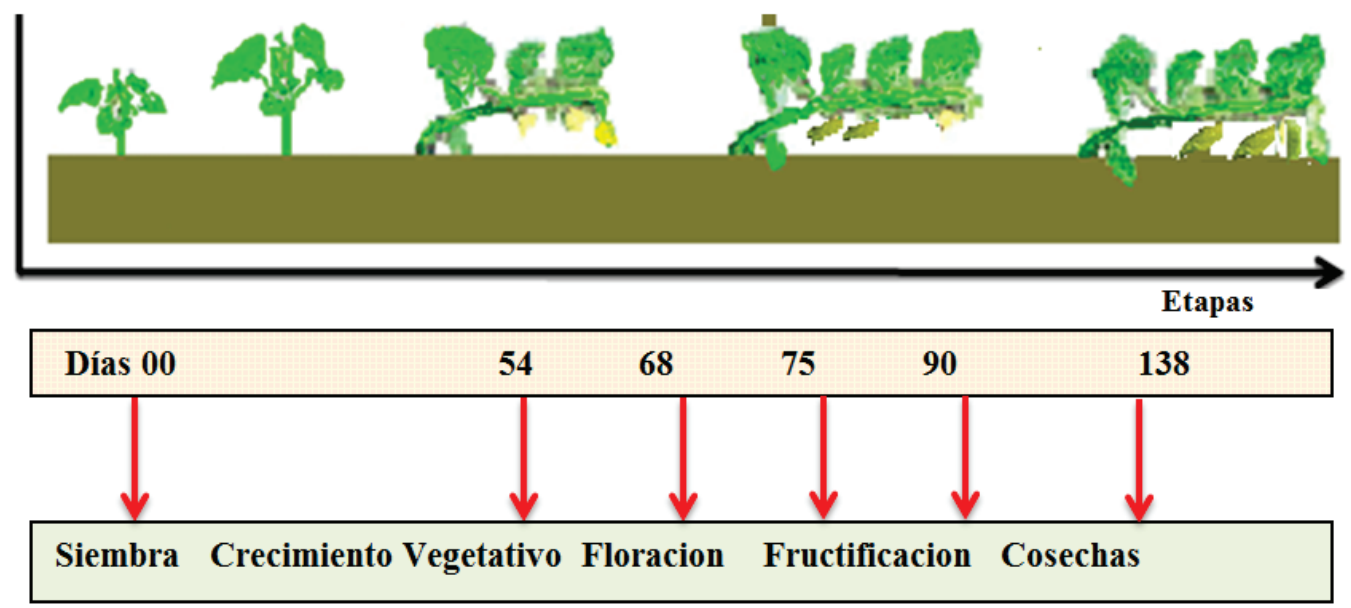

Aplicaciones 20 días 60 días

Figura 1. Aplicación de dosis en la etapa de crecimiento y floración.

\section{RESULTADOS}

\section{Cantidad de frutos por planta}

El análisis estadistico no encontró diferencias significativas entre los tratamientos $(p \leq 0,05)$, lo que se interpreta que las dosis de citoquinina (Antesis Plus) no influyeron en los tratamientos. Los resultados se pueden apreciar en la Tabla 2.

\begin{tabular}{cccc} 
Tratamiento & $\begin{array}{c}\text { Dosis de Citoquinina } \\
(\mathrm{m} / / 2 \text { L de agua) }\end{array}$ & $\begin{array}{c}\text { Cantidad de } \\
\text { caigua }\left(\mathbf{N}^{\circ}\right)\end{array}$ & $\begin{array}{c}\text { Duncan } \\
\text { Agrupamiento }\end{array}$ \\
\hline $\mathrm{T}_{3}$ & 100 & 26,0 & $\mathrm{a}$ \\
$\mathrm{T}_{4}$ & 150 & 24,0 & $\mathrm{a}$ \\
$\mathrm{T}_{2}$ & 50 & 23,0 & $\mathrm{a}$ \\
$\mathrm{T}_{5}$ & 200 & 22,0 & $\mathrm{a}$ \\
$\mathrm{T}_{1}$ & 0 & 19,0 & $\mathrm{a}$ \\
\hline
\end{tabular}

Nota: Promedio con la misma letra son estadísticamente homogéneos. 


\section{Peso de frutos por planta}

Respecto al peso de fruto por planta, se obtuvo significativo mediante el análisis de varianza al $5 \%$, esto se muestra en la Tabla 3 . El resultado quiere decir que influyó la dosis de citoquinina lo que es conveniente en el rendimiento.

\section{Peso de un fruto por tratamiento}

Respecto al peso de un fruto representativo, se determinó con el análisis de varianza al $5 \%$ que son significativos, lo que se interpreta que influyó la dosis de citoquininas, siendo favorable en calidad (Ver tabla 3),

También se expone la calificación de los promedios obtenidos por la operación de la
Prueba de Duncan al $5 \%$ que define el $\mathrm{T}_{3}$ con $112,15 \mathrm{~g}$ destacó en relación a los demás tratamientos, siendo favorable para el agricultor.

\section{Rendimiento comercial}

En cuanto al rendimiento por hectárea se determinó por medio del análisis de varianza al $5 \%$, que es signifcativo esto quiere decir que influyó la dosis de citoquinina, siendo este resultado beneficioso en lo comercial.

Asimismo se obtuvo los resultados con la Prueba de Duncan al $5 \%$, que se observa en la Tabla 3, que indica que hay variación de los promedios, destacándose el $T_{3}$ con 30,60 tn/ha en relación a los demás.

Tabla 3. Tratamientos, dosis, peso y rendimiento comercial en el cultivo de caigua

\begin{tabular}{ccccc} 
Tratamiento & $\begin{array}{c}\text { Dosis de Citoquinina } \\
(\mathrm{ml} / 2 \text { L de agua) }\end{array}$ & $\begin{array}{c}\text { Peso de frutos } \\
\text { por planta }(\mathrm{kg})\end{array}$ & $\begin{array}{c}\text { Peso de un } \\
\text { fruto }(\mathrm{g})\end{array}$ & $\begin{array}{c}\text { Rendimiento } \\
\text { comercial (tn/ha) }\end{array}$ \\
\hline $\mathrm{T}_{3}$ & 100 & $2,2768 \mathrm{a}$ & $112,155 \mathrm{a}$ & $30,600 \mathrm{a}$ \\
$\mathrm{T}_{4}$ & 150 & $1,8498 \mathrm{~b}$ & 102,018 & $25,492 \mathrm{bc}$ \\
$\mathrm{T}_{2}$ & 50 & $1,6308 \mathrm{bc}$ & $88,76 \mathrm{~b}$ & $26,453 \mathrm{~b}$ \\
$\mathrm{~T}_{5}$ & 200 & $1,451 \mathrm{dc}$ & $94,288 \mathrm{ab}$ & $22,011 \mathrm{dc}$ \\
$\mathrm{T}_{1}$ & 0 & $1,1698 \mathrm{~d}$ & $81,933 \mathrm{~b}$ & $19,522 \mathrm{~d}$ \\
\hline
\end{tabular}

Nota: Promedio con la misma letra son estadísticamente homogéneos

\section{Análisis foliar}

El estudio de análisis foliar muestra como influyó los elementos con relación a la dosis de citoquinina en $100 \mathrm{~g}$ de materia seca de macronutrientes y en $\mathrm{mg} / 1000 \mathrm{~g}(\mathrm{ppm}) \mathrm{de}$ micronutrientes, esto se aprecia en la Tabla 4, que indica el Tratamiento 3 se muestra las condiciones normales en la mayoría de los nutrientes; sin embargo el macronutriente potasio obtuvo baja calificación. Por lo tanto a esta concentración de elementos influyó en el mayor rendimiento.

Tabla 4. Análisis foliar, según la dosis de aplicación de Antesis Plus

\begin{tabular}{|c|c|c|c|c|c|c|c|c|c|c|c|}
\hline & \multicolumn{2}{|c|}{$\mathrm{T}_{1}$} & \multicolumn{2}{|c|}{$\mathrm{T}_{2}$} & \multicolumn{2}{|c|}{$\mathrm{T}_{3}$} & \multicolumn{2}{|c|}{$\mathrm{T}_{4}$} & \multicolumn{2}{|c|}{$T_{5}$} & \multirow[b]{2}{*}{$\begin{array}{l}\text { Valores } \\
\text { Normales }\end{array}$} \\
\hline Elementos & $\begin{array}{c}0 \mathrm{ml} \\
200 \mathrm{~L} \\
\mathrm{H}_{2} \mathrm{O}\end{array}$ & Calific, & $\begin{array}{c}50 \mathrm{ml} \\
200 \mathrm{~L} \\
\mathrm{H}_{2} \mathrm{O}\end{array}$ & Calific, & $\begin{array}{c}100 \mathrm{ml} \\
200 \mathrm{~L} \\
\mathrm{H}_{2} \mathrm{O}\end{array}$ & Calific, & $\begin{array}{c}150 \mathrm{ml} \\
200 \mathrm{~L} \\
\mathrm{H}_{2} \mathrm{O}\end{array}$ & Calific, & $\begin{array}{c}200 \mathrm{ml} \\
200 \mathrm{~L} \\
\mathrm{H}_{2} \mathrm{O}\end{array}$ & Calific, & \\
\hline $\mathrm{N} \%$ & 2,80 & Bajo & 3,60 & Normal & 4,80 & Normal & 5,80 & Alto & 6,54 & Alto & $3,50-5,50$ \\
\hline P \% & 0,39 & Normal & 0,42 & Normal & 0,60 & Normal & 0,25 & Bajo & 0,91 & Alto & $0,30-0,70$ \\
\hline $\mathrm{K} \%$ & 3,00 & Normal & 2,80 & Normal & 6,20 & Bajo & 6,80 & Alto & 2,00 & Bajo & $2,50-6,00$ \\
\hline Ca \% & 1,15 & Bajo & 1,20 & Вајо & 4,25 & Normal & 5,20 & Alto & 5,10 & Alto & $1,25-5,00$ \\
\hline Mg\% & 0,70 & Normal & 0,65 & Normal & 1,48 & Normal & 1,80 & Alto & 1,55 & Alto & $0,60-1,50$ \\
\hline $\mathrm{Na} \%$ & 0,01 & Normal & 0,05 & Normal & 0,12 & Normal & 0,21 & Alto & 0,15 & Normal & $0,01-0,20$ \\
\hline Cu ppm & 27 & Alto & 15 & Normal & 22 & Normal & 25 & Normal & 33 & Alto & $10-25$ \\
\hline Fe ppm & 85 & Normal & 45 & Bajo & 140 & Normal & 190 & Normal & 216 & Alto & $50-200$ \\
\hline Zn ppm & 25 & Bajo & 32 & Normal & 51 & Normal & 65 & Normal & 75 & Alto & $30-70$ \\
\hline B ppm & 10 & Bajo & 23 & Bajo & 78 & Normal & 20 & Bajo & 62 & Normal & $25-8 / 0$ \\
\hline Rdto ha & 17,84 & & 25,79 & & 31,04 & & 25,95 & & 21,76 & & \\
\hline
\end{tabular}

Fuente: INIA (2016) Instituto Nacional de Innovación Agraria Huaral.

Nota: Cabe mencionar que en el análisis foliar se determinó la concentración de los elementos, de acuerdo a la dosis de citoquinina. 


\section{DISCUSIÓN}

En la cantidad de frutos por planta no hubo efecto de dosis de citoquinina; sin embargo se obtuvo mayor cantidad en el tratamiento 3 lo cual fue favorable; puesto que esta dosis influyó en las reacciones biquimicas obteniéndose desarrollo del fruto y mayor cantidad, lo analizado se sostiene con Wardle, Reynolds, Zurowski y Looney (1992), que la citoquinina al ser aplicadas en forma exógena a las plantas, estimula una serie de procesos fisiológicos, metabólicos y de desarrollo, la principal respuesta corresponde a la división y elongación celular a través de un incremento de las paredes celulares (Ver Tabla 2).

Los mayores rendimientos en peso de frutos por planta, fueron observados en el tratamiento 3 , Estos resultados sugieren que hubo efecto de la dosis, es decir intervino en la división celular por ende en el desarrollo de los órganos de la planta y la translocación de carbohidratos esto se relaciona con Antesis Group (2019), menciona que la citoquinina (Antesis Plus), se utiliza en la floración y cuajado, favoreciendo el crecimiento vegetal al mismo tiempo que induce la floración, fructificación y engorde del fruto (Ver Tabla 3).

En rendimiento comercial que se indica en la Tabla 3 , indica que hubo efecto de dosis, es decir no hay homogeneidad estadística. Asimismo el tratamiento 3 obtuvo el mayor rendimiento, lo que se interpreta que a dosis adecuada de citoquinina influyó en el desarrollo de ramas, flores, fructificación, favoreciendo en el rendimiento, esto se contrasta con Villatoro (2014), que evaluó diferentes dosis de CPPU (Citoquinina) a 25, 50, 100 y 150 ppm, aplicándose en la floración y cuajado lo que determinó alcanzó a 27,61 tm/ha a 100 ppm y disminuyó a 27,47 tm/ ha a 150 ppm.

En cuanto al peso promedio de un fruto, que se aprecia en la Tabla 3, señala que el tratamiento 3 obtuvo el mayor peso, pero no influyó significativamnte, sin embargo a esta dosis favoreció en la la presencia, vistosidad y peso, es decir en la calidad de fruto, este resultado se fundamenta con Antesis Group (2019), menciona que Antesis Plus (citoquinina), se utiliza en la floración y cuajado, favoreciendo el crecimiento vegetal al mismo tiempo que induce la floración, fructificación y engorde del fruto.

En análisis foliar destacó el tratamiento 3 que obtuvo la mayoría de los elementos en la calificación normal con excepcion de potasio en $\mathrm{g} / 100 \mathrm{~g}$ de materia seca; puesto que a esta concentración resultó buen rendimiento comercial con 30,60 tn /ha, por lo tanto a esta dosis de citoquinina fue favorable en la disponibilidad de absorción de macro y micro elementos, ya que contribuyeron en el rendimiento (Ver Tabla 4).
Los resultados del presente estudio muestran que el mayor rendimiento de caigua obtuvo el tratamiento 3 con la dosis de $100 \mathrm{ml}$ por $200 \mathrm{~L}$ de citoquinina (Antesis Plus), siendo favorable para los agricultores de la zona, respecto a las evaluaciones realizado en campo como y en laboratorio: Cantidad de frutos por planta, peso de frutos por planta y peso de un fruto por planta sobresalió el tratamiento 3 , lo cual es favorable en la calidad.

En análisis económico se determinó que la mayor utilidad obtuvo el tratamiento 3 con S/, 11695 Nuevos Soles diferenciándose al (testigo) $T_{1}$ con S/, 3993 Nuevos Soles, lo que es beneficioso.

\section{AGRADECIMIENTOS}

Los autores de la presente investigación agradecen a la comunidad de Santa Elena de la provincia de Barranca, Universidad Nacional Santiago Antunez De Mayolo, Universidad Nacional Jose Faustino Sánchez Carrión y al Instituo Nacional de Imnovacion Agraria - Huaral por su apoyo tecnico y su prestación de Servicios de Laboratorio lo que hiceron posible este trabajo de investigación

\section{REFERENCIAS BIBLIOGRAFÍCAS}

Antesis group (2019). Antesis group, S.A.C. productos para agricultura confianza y c a I i d a d p a r c u I t i vos. http://www.antesisgroup.com

Arohuanca, S, (2015). Efecto de aplicación de citoquinina en rendimiento de dos variedades de vainita (Phaseolus vulgaris $L$,) En el Distrito de Samegua - Moquegua. Tesis para Optar el título profesional. Universidad José Carlos Mariátegui, Moquegua.

Bahamonde, P. (2006). Efecto de la aplicación de reguladores de crecimiento sobre híbridos de Calas (Zantedeschia spp.). Tesis para Optar al Grado de Licenciado en Agronomía. Universidad Austral de Chile. Valdivia,Chile.

Cadenas, M, (2015). Evaluación de citoquinina en Esquejes de Penstemon (Penstemon campanulatus); villa canales, Guatemala. Tesis para Optar el Título profesional. Universidad Rafael Landívar. Guatemala.

Campos, M. \& Quintana, N., (2013) Tratamiento con ensalada de caigua (Cyclanthera pedata) a mujeres adultas con hipertrigliceridemia en el caserío Santa Rosa, distrito de Lurín (Lima), 2011. Tesis para Optar al Título Profesional de Químico Farmacéutico. Universidad Wiener, LimaPerú. 
INIA (2016). Hoja de Análisis Básico de Fertilidad y Análisis de Tejido Vegetal. Estación Experimental Agraria Donoso Kiyotada Miyagawa,-Huaral-Perú.

Salisbury, F. \& Ross, C. (1994). Fisiología vegetal. (cuarta edición). México D. F.: Editorial Iberoamericana.

Villatoro, E. (2014). "Efecto de citoquinina (CPPU) sobre el cuaje y Rendimiento en el cultivo de Minisandia (Cytrullus lannatus), Cucurbitácea; Estanzuela, Zacapa. Trabajo para optar el título de Ingeniero. Universidad Rafael Landívar, Guatemala.

Wardle, D. Reynolds, A. Zurowski, C. \& Looney, N.
(1992). Phenylureas CPPU and thidiazuron affect yield components, fruit composition, and storage potential of four seedless grape selections. Journal of the American Society for Horticultural Science, 117(1), 85-89.

\section{Correo electrónico:}

jesusila_82_21@hotmail,com

Revisión de pares:

Recibido: 25/10/2019

Aceptado: 20/12/2019 